\title{
Aportes del racionalismo crítico de K. Popper a la filosofia de las ciencias sociales.
}

\author{
Contributions of K. Popper's critical rationalism to the social sciences \\ philosophy.
}

\section{Roger de Jesús Sepúlveda Fernández ${ }^{1}$}

Sepúlveda F. Roger. miradas N¹3 - 2015. ISSN: 0122 994X Págs 160 - 176

Recepción: Mayo 14 de 2015

Aprobación: Septiembre 23 de 2015

Publicación: Diciembre 17 de 2015

\section{Resumen}

En este artículo se desarrolla tres concepciones de teoría en K Popper, basado en una visión biológica y evolutiva del conocimiento; luego, se examinan estas nociones en el contexto de la filosofía en las ciencias sociales; finalmente, se valora cómo aporta a la discusión de la naturaleza de las teorías en administración, especialmente en la literatura especializada colombiana de la administración.

Palabras Clave: Ciencia, teoría, lógica de la situación, ciencias sociales, administración.

\begin{abstract}
In this article three conceptions of theory in K. Popper are developed, based on a biological and evolutionary view of knowledge; then, these notions are discussed in the context of philosophy in social sciences; finally, it evaluates how it contributes to the discussion of the nature of theories in administration, especially in the specialized Colombian literature of administration.
\end{abstract}

Key Words: science, theory, logic of the situation, social science, administration.

1 Sepúlveda Fernández, R. de J. (2012). Estructuras teóricas de la administración ¿Ciencia o tecnología? Un enfoque filosófico. Tesis de Maestría en Filosofía, Universidad de Caldas: Manizales, Colombia.

rogersepulveda@mail.uniatlantico.edu.co 


\section{Introducción.}

La epistemología o la lógica de la investigación científica, según Popper (1962:48-49) es un área de la filosofía de la ciencia que realiza un análisis crítico de la investigación científica, especialmente la noción de ciencia, el lenguaje de la ciencia, los criterios con que se demarcan concluyentemente lo científico y lo no científico, la noción de teoría, el progreso científico, la racionalidad del progreso científico, las reglas metodológicas de la investigación científica, entre otros problemas epistemológicos en ciencia. En cuanto al estudio de la filosofía de las ciencias sociales las tesis de Popper "abren interesantes perspectivas de investigación; ya sea para admitirlas, revisarlas o refutarlas" (Gómez; 2003: 59). A continuación se consideran algunos tópicos analizadas por Popper que son significativas para el estudio de las ciencias sociales.

\section{Noción de teoría.}

Popper (1992:309) desarrolla una noción de teoría a partir de una concepción biológica y evolutiva del conocimiento humano, basado en algunos planteamientos de Darwin, con la que explica la existencia de diferentes estructuras en los seres vivos, con que estos resuelven los problemas de adaptación a su entorno natural, ambiental y social.

La teoría evolutiva darwinista asumida por Popper es una explicación de la adaptación de los animales a su entorno. Este proceso de adaptación Darwin lo explica desde la noción de selección natural de las especies y en la especialización de sus órganos basado en el perfeccionamiento del funcionamiento con el que el animal soluciona las dificultades y problemas con mayor velocidad, habilidad e inteligencia que otras especies. Lo que Darwin mostró son los mecanismos de selección natural con que los animales pueden simular, en principio, el acto creador en la resolución de sus problemas. En este sentido, en el proceso evolutivo de las especies emergen estructuras genéticas, y en el animal humano las estructuras simbólicas con que resuelve sus dificultades.

"Uno de los puntos claves del darwinismo es que solo es probable que sobreviva, de hecho, el organismo que muestre en su comportamiento una tendencia, disposición o propensión fuerte a luchar por su supervivencia; tal disposición tenderá a desarrollar una estructura genética con la que se adapta al medio... las otras estructuras simbólicas, entre las que destaca las teorías científicas como estructuras simbólicas cognoscitivas]es un intento de explicar los cambios genéticos que conducen a una adaptación y al desarrollo de mejores habilidades para la supervivencia" (Popper, 2007:315-319).

El filósofo argumenta que los organismos, especialmente el humano, en los procesos evolutivos bilógicos y culturales ha desarrollado dos tipos de estructuras: las genéticas y las cognoscitivas.

\section{Estructuras genéticas.}

Popper (2007:309), apoyado en la teoría evolutiva de Darwin, argumenta que todos los animales, "Desde la ameba hasta Einstein", han desarrollado varias clases de estructuras genéticas (biofísico-químicas) portadoras de información, las cuales son producto de la evolución biológica y que los individuos reciben por pertenecer a una especie determinada. 
Estas estructuras genéticas orientan en los seres vivos el desarrollo de los órganos, disposiciones biológicas y ciertos tipos de comportamientos involuntarios e inconscientes con que solucionan problemas relacionados con la supervivencia, la adaptación o la transformación del entorno natural, ambiental y sociocultural.

En el árbol de la evolución del conocimiento crecen ramas a partir de un tronco común. Este tronco está formado por nuestros ancestros unicelulares, comunes a todos los organismos vivos... Todo animal ha nacido con expectativas o anticipaciones adquiridas (heredadas) que pueden tomarse como una especie de conocimiento innato [herencia] con el cual soluciona sus problemas. (Popper, 2007:306-310).

El austriaco argumenta que algunos seres vivos, no solo poseen estructuras genéticas que controlan el funcionamiento embrionario y biológico de sus órganos; sino que, además, han desarrollado otras estructuras simbólicas cognoscitivas con que desarrolla habilidades especializadas y artefactos ejecutivas para actuar en el entorno social y cultural.

En muchos organismos se pueden distinguir, al menos, dos estructuras distintas: una que controla las conductas del organismo...y otra con las cuales las ejecuta... Por ejemplo, los genes de la especie humana controlan el desarrollo embrionario del ojo, lo mismo que la curiosidad visual. ... mientras que con las estructuras relacionadas con el universo abstracto de significados realiza la actividad racional de la conciencia y del comportamiento humano. (Popper, 2007:322,275).

Con la tesis evolutiva de las estructuras,
Popper explica dos problemas: la tensión entre la ortogénesis versus mutación accidental $^{1}$ y el problema de la influencia del universo simbólico abstracto en la conciencia y en la conducta racional humana en contexto social y cultural.

\section{Las estructuras cognoscitivas.}

Elfilósofo sustenta, desde la teoría evolutiva, que los humanos han desarrollo, no solo las estructuras biológicas de disposición central, coordinadas por cerebro, que regulan el funcionamiento del sistema nervioso, sino otro tipo de estructuras: las cognoscitivas. Las estructuras biológicas de disposición central coordinadas por cerebro posibilita en el humano el desarrollo estructuras cognoscitivas basado en las funciones neurofisiológicas y en las funciones del lenguaje humano (tales como la expresiva, señaladora, descriptiva y argumentativa). Las estructuras neurobio-fisiológicas que son coordinadas por el cerebro, no solo permiten funciones vitales en el ser humano, sino que también es el centro de la creación de estructuras simbólicas cognoscitivas que son la génesis del pensamiento y la cultura humana.

Popper clasifica las estructuras cognoscitivas en dos tipos: las subjetivas o tácitas (sentido común) y las explícitas u objetivas. Las estructuras cognoscitivas subjetivas $^{2}$ emergen en el sujeto de forma inconsciente con la adquisición del lenguaje humano y con los procesos de inserción sociocultural a lo largo de su vida, especialmente en la infancia. Estas estructuras son la base del conocimiento tácito o subjetivo que se conquista por la adquisición del lenguaje nativo o por vía de la experticia directa con las cosas e influencia de los procesos socioculturales.

Las estructuras cognoscitivas objetivas, por el contrario, están relacionadas con la capacidad humana de la conciencia ${ }^{3}$ de crear artefactos exosomáticos, los cuales se 
expresan en múltiples formas como son la técnica, el arte, la filosofía, la ciencia y las humanidades. Estas estructuras permiten crear destrezas y habilidades mentales técnicas, ejecutivas y automáticas. Las estructuras cognitivas objetivas posibilitan la creación de mundos subjetivos culturales $\mathrm{y}$ sociales humanos que sobrepasan lo meramente natural. Popper elabora una teoría tripartita del mundo para explicar el proceso creativo exo-somático en los humanos.

\section{Visión ontológica tripartita de la realidad del mundo en Popper.}

Popper (1994) concibe mundo o realidad ${ }^{4}$ ontológicamente ${ }^{5}$ como el producto de la evolución de la naturaleza y de la creación de los seres vivos, especialmente la humana. Esta realidad está compuesta por tres mundos: ${ }^{6}$

El mundo de las cosas físicas: son los cuerpos animados e inanimados, los objetos sólidos, el aire, el agua, los acontecimientos, las fuerzas de atracción magnética y eléctrica, la gravedad, el movimiento, el frio, el calor. Estos cuerpos físicos son unos productos de la evolución de la naturaleza y otros de la creación humana. A este mundo pertenece el mundo natural y el mundo de los artefactos físicos creados por el hombre.

El mundo de las experiencias subjetivas: Está compuesto de las experiencias conscientes e inconscientes de la conciencia humana. Este mundo emerge en el sujeto desde la estructura biológica (cerebral), de la estructura del lenguaje y de las interacciones con el entorno sociocultural y ecológico.

El mundo de los productos de la mente humana. En este mundo se incluyen las obras de creación humana objetivados en los libros, las sinfonías, las obras de arte, las teorías científicas, los artefactos tecnológicos (máquinas, utensilios, técnicas, artesanías) y artefactos sociales (instituciones y organizaciones), así como los productos planeados y deliberados por la actividad mental humana expresados objetivamente en artefactos lingüísticos; y todas las creaciones del hombre que hacen parte del mundo uno. El mundo tres incide o transforma el mundo uno, el dos y el mismo mundo tres. Popper (2007:275) en el universo simbólico del mundo tres, incluye las promesas, los fines, los diversos tipos de reglas gramaticales, las reglas de urbanidad y las reglas lógicas; así como las reglas de juego (ajedrez, fútbol), de justicia, artísticas, técnicas y científicas.

El mundo tres es la base de la cultura con la cual la especie humana soluciona los problemas y errores, y supera las soluciones biológico-naturales. Con la creación del lenguaje surgieron los sistemas numéricos y teóricos, las instituciones y otros lenguajes de simbolización de la realidad que tienen un efecto en la vida humana y social.

Los tres mundos están interconectados entre sí e interactúan de forma tal que a veces se ocultan entre ellos parcialmente. El problema que valora Popper (1994:275) es la influencia del universo de los significados abstractos del mundo tres (teorías) sobre la conducta (mundo dos) y el universo físico (mundo uno).

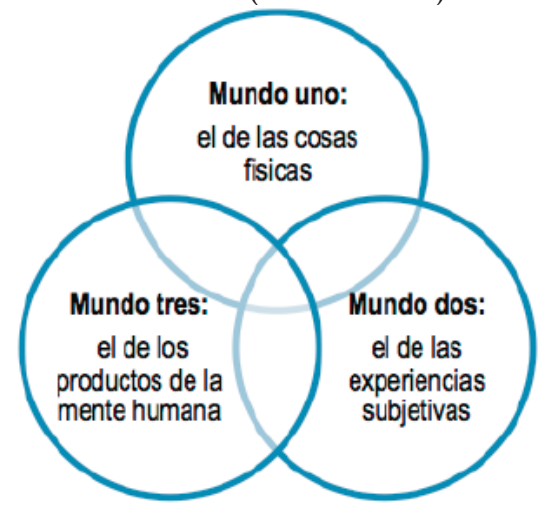

Gráfica 1. Teoría de los tres mundos de Popper. Fuente: Propia. 
Concepción de teoría científica como estructuras simbólicas cognoscitivas objetivas.

¿Representan las teorías la estructura de la realidad del mundo, o son modelos que interpretan con éxito los fenómenos del mundo uno, dos y tres? La respuesta a esta pregunta es una crítica a la noción realista o esencialista de la teoría del positivismo lógico, que concibe las teorías como estructuras que representan el funcionamiento regular del mundo natural.

Popper rechaza el esencialismo ${ }^{7}$ teórico y el realismo ontológico del neopositivismo lógico porque define que las teorías no son explicaciones últimas de los fenómenos y ni representan las propiedades esenciales de la estructura del mundo natural, sino que es una interpretación que el investigador conjetura que así funciona el mundo. La teoría para Popper es una red simbólica que inventa para interpretar el funcionamiento del mundo natural, social y humano. "Las teorías de la naturaleza las pensamos más bien como conjeturas que describen regularidades (conjeturales) de las propiedades estructurales de la naturaleza o de nuestro propio mundo. Aquí está [dice Popper] la diferencia y semejanza entre mi punto de vista y el esencialismo" (Popper, 1994: 237). En otra parte expresa: "Una teoría es una estructura o esquema racional de ideas que describen, explican ${ }^{8}$ e interpretan el comportamiento regular [e irregular] de los fenómenos. Una teoría es una hipótesis (proposiciones cuya verdad es una conjetura) o suposición" (Popper, 2007:25,39-47).

Según Popper (1994:60), las teorías del sentido común y las objetivas son invenciones libres del intelecto humano, que intentan imponerse a la naturaleza para explicar su funcionamiento, pero que raras veces tienen éxito. Las teorías son respuestas que inventa el ser humano como forma de sobrevivencia, porque si las hipótesis o conjeturas fracasan el humano no muere y sobrevive, a diferencia de otros organismos vivos que mueren cuando fracasan las respuestas que ellos elaboran con los mecanismos o estructuras biológicas.

Las teorías son conjeturas son respuestas audaces que los científicos inventan para interpretar $^{9}$ el funcionamiento del mundo, las cuales son refutables racionalmente cuando entran en conflicto con las observaciones. Además, son modelos aproximados que simulan simbólicamente el funcionamiento de los fenómenos y orientan las observaciones de los mismos. Por lo tanto, las teorías ${ }^{10}$ o estructuras cognoscitivas objetivas son producto de la capacidad simbólica del ser humano, y emergen de las funciones del lenguaje con los que crea universos mitológicos, metafísicos, científicos, técnicos, entre otros.

Las teorías científicas son, como todas las representaciones, sistemas de enunciados expresados en fórmulas o esqueletos simbólicos. Las teorías son redes que el investigador crea para apresar aquello que llamamos el mundo: para racionalizarlo, explicarlo y dominarlo. En ciencia siempre se busca que la malla sea cada vez más fina. (Popper, 1962:57).

Las teorías están compuestas de premisas que se deducen de enunciados universales y singulares. Los enunciados sintéticos se clasifican en estrictamente universales y numéricamente universales. Con ellos el investigador busca expresar la estructura del mundo a través de teorías y leyes basadas en el principio de la causalidad.

Las teorías son principios heurísticos que el investigador crea sostenido en nociones 
filosóficas (epistemológica, ontológicas, metodológicas y Praxeológicas) tácitas o explícitas con que observa el mundo.

Popper en el desarrollo de la obra propone diferentes nociones de teorías una fuerte o clásica, moderada y plástica.

La concepción fuerte de teoría la desarrolla en Lógica de la investigación científica, entre la década de los años treinta a la de los sesenta del siglo XX, a partir de la lógica, como instrumento fundamental para sustentar racionalmente las teorías y la actividad científica. Popper clasifica las teorías en empíricas, que incluyen conceptos ostensivos (conceptos universales y singulares $^{11}$ y de clase); enunciados analíticos, sintéticos ${ }^{12}$ y existenciales. $^{13}$

El científico que se ocupa con una investigación determinada, digamos de la física, puede atacar su problema de modo directo: puede dirigirse al corazón del asunto, esto es, al corazón de una estructura organizada. Pues existe una estructura en las doctrinas científicas. (Popper, 1962:14).

Las teorías científicas son afirmaciones verdaderas y universales que describen la estructura del mundo (no importa el espacio-temporal). Las nuevas teorías no son ad hoc ni circulares con relación a las anteriores; no se limitan a repetir el explicandum, y más bien lo corrigen o contradicen.

Popper propone que las explicaciones teóricas (explicans) son satisfactorias si cumplen los siguientes requisitos:

$1.1 \mathrm{La}$ cuestión o problema (explicandum) se debe explicar lógicamente;
1.2 Las explicaciones ${ }^{14}$ (explicans) han de ser verdaderas, aunque en general no se sepa; no han de ser falsas sino hasta después del examen más crítico. Si no se sabe que es verdadero (lo más probable) se ha de disponer de elementos de juicio independientes ${ }^{15}$ a favor suyo; es decir, "juicios contrastables o falsables con contenidos distintos a los de la explicación que permitan interpretar el explicandum como un caso particular de un efecto reproducible" (Popper, 2007:233);

1.3 Desde lo metodológico, las teorías son satisfactorias porque logran integrar con coherencia la riqueza del contenido, la simplicidad, la belleza y la imaginación o ingenio $^{16}$ del investigador.

Popper "clasifica las teorías por el grado de preferencia en teóricas (por ser verdaderas) y pragmáticas (porque orientan la acción)". "El hombre de acción elige entre varias alternativas concretas, un conjunto de expectativas; es decir, de teorías sobre el mundo que exigen una actitud racional pragmática. ¿Qué teorías elegirá desde el punto de vista racional el hombre de acción práctica? ...la mejor contrastada como base de acción" (2007:29,38).

Este criterio ha sido valorado por los científicos sociales de sensato e innovador en comparación con las tesis de los neopositivistas porque proporciona un estatuto epistémico y ontológico a las teorías en tanto que permite evaluar la naturaleza de las teorías en las ciencias sociales.

La concepción moderada de teoría Popper la desarrolla desde la noción de red $\mathrm{o}$ modelos ${ }^{17}$ teóricos que tiene una estructura diferente a una estructura lógico-matemática. Las teorías son redes o modelos inventadas por el ser humano 
para explicar los fenómenos del mundo, las cuales tienen niveles de universalidad, grados de precisión, poder de simplicidad mejor que las existentes, y capacidad de ser refutables racionalmente.

En filosofía de la ciencia, los modelos representan o interpretan las teorías en cinco posibles formas:

$\checkmark$ Modelos lógicos: son las interpretaciones semánticas de un sistemas de axiomas (Ej. el cálculo de una teoría).

\section{$\checkmark$ Modelos matemáticos: son representaciones aritméticas de las teorías empíricas; es decir, son un conjunto de proposiciones matemáticas que tienen la misma forma de las leyes de la teoría que representa $(\mathrm{Ej}$. isomorfismo estructural).}

\section{$\checkmark$ Modelos}

son representaciones

analógicos:

tridimensionales de un objeto $\mathrm{o}$ sistema de forma gráfica (Ej. el sistema planetario). Los Modelos son analogías o metáforas ontológicas que sustentan creencias y concepciones metafísicas del mundo que simbolizan e interpretan la realidad.

Para T. Kuhn el descubrimiento científico es un proceso complejo que involucra el reconocimiento, tanto de lo que existe como de lo que es o no es, "...con formas de ver (Gestalt) que articulan creencias, preconcepciones del mundo... a través de estructuras que dirigen, cohesionan y modelan prácticas investigativas en una comunidad" (Kuhn,1992). En este sentido un modelo es una estructura o rompecabezas que simboliza una parte de la realidad y orienta la solución de problemas teóricos o prácticos en un saber determinado.

$\checkmark$ Modelos teóricos: son las representaciones simbólicas de un objeto o fenómeno que permite atribuirle una estructura interna, y están sustentados en una teoría (Ej. el modelo del átomo de Bohr).

\section{Modelos imaginarios: son} criterios heurísticos para explicar el funcionamiento de los objetos o sistemas con ciertos principios y condiciones $(\mathrm{Ej}$. el campo magnético de Maxwell).

La tercera noción de teoría en Popper está asociada a hipótesis, conjeturas o expectativas definidas como artefacto simbólico exosomático de control plástico (sistemas plásticos peircianos) los cuales orienta a los seres humanos en la eliminación de errores y en la solución de problemas en su entorno natural, social, humano y ecológico. Considera, además, las teorías como redes cognoscitivas de control plástico que coordinan y orientan las acciones ejecutivas humanas. Estas nociones de teoría son polémicas, pero abre perspectivas filosóficas en la forma de interpretar el mundo social, humano y ambiental que desde la visión fuerte de teoría de los neopositivistas son excluidos. El Problema está en definir ¿qué es lo que hace científico a una teoría? ¿O será más bien que la práctica científica, especialmente en lo social, no se agota en la creación de teorías objetivas?.

\section{Objeto de las ciencias.}

Popper valora dos tipos de conocimiento: el conocimiento del sentido común y el conocimiento científico, que lo clasifica en teórico, experimental y práctico. Las ciencias, sean teóricas, empíricas o prácticas, son lenguajes simbólicos cognoscitivos especializados compuestos por sistemas teóricos y metodológicos basado en visiones filosóficas [metafísicas, ontológicas, epistémicas y Praxeológicas] que orientan la búsqueda de soluciones a problemas en cualquiera de los tres mundos antes definidos. 
La ciencia es una práctica sociocultural cognoscitiva basada en una racionalidad crítica cuyo fin es proponer teorías objetivas con la capacidad interpretar el mundo con profundidad, precisión, universalidad, utilidad y satisfacción.

\section{El objetivo de la ciencia es dar explicaciones $^{18} \quad$ satisfactorias... verdaderas o que se supone que lo son, ya que carece de interés pedir explicaciones de algo imaginario... La ciencia es creación del pensamiento humano y no es solo el resultado de la aplicación de un método experimental o de la lógica inductiva. (Popper, 1994:59,231- 232).}

Las ciencias son sistemas de meta-reglas inventadas por los investigadores con el fin de encontrar soluciones críticas a los problemas que enfrenta. Esas soluciones no solo exige invenciones de estructuras teóricas, sino que también proponen métodos $^{19}$ y formas de conocimiento científico.

"La intencionalidad del investigador es crear teorías explicativas objetivas que describan ciertas propiedades estructurales del mundo que permitan deducir, de ciertas condiciones iniciales, los efectos que tratan explicar" (Popper, 1992:59).

\section{El progreso del conocimiento científico.}

El progreso de las ciencias está asociado a la crítica racional. La ciencia progresa cuando los científicos desarrollan una actividad crítica en la práctica investigativa, especialmente, porque crean "teorías que tienen mayor contenido explicativo, niveles más elevados de universalidad, grados de precisión y criterios de simplicidad cada vez más exitosos" (Popper, 1992:234) con el fin de reemplazar a sus predecesoras menos adecuadas.
Las nuevas teorías son un intento de explicación satisfactoria y exitosa ${ }^{20}$, mejor que las explicaciones existentes, en la medida en que proponen mejores criterios para valorar críticamente las problemáticas y soluciones del mundo natural o social investigado. "Sostengo que toda explicación puede ser hecha a su vez mediante una teoría o conjetura de un grado superior de universalidad que las anteriores.... El progreso del conocimiento científico consistirá en corregir y modificar el conocimiento previo" (Popper, 1992:236,306).

Según Popper (1994:71), la respuesta correcta de cómo se da el progreso del conocimiento científico puede sustentarse desde el racionalismo crítico, que es una actitud filosófica de crítica permanente a nuestras ideas científicas; actitud que nace con la filosofía griega.

La función argumentadora no sólo es la más elevada de las cuatro funciones del lenguaje humano... sino que es la última en aparecer en el proceso evolutivo... ella está asociada al desarrollo de los discursos científicos disciplinares... Podemos decir que la función argumentadora del lenguaje ha creado el instrumento más poderoso de adaptación biológica que haya surgido en el transcurso de la evolución humana: la ciencia y la técnica... y, al igual que las demás funciones, se ha desarrollado mediante el método del ensayo y el error. (Popper, 2007:280-285).

Las teorías son estructuras cognitivas objetivas que cumplen los siguientes requisitos:

1. Son enunciados universales que interpretan la singularidad y aparente irregularidad del mundo. 
2. No son meras recopilaciones de observaciones sobre el mundo, sino que las observaciones se interpretan a la luz de las teorías, la observación siempre es selectiva.

3. El grado de verdad, mensurabilidad o exactitud de las teorías depende de los enunciados observacionales.

\section{Las teorías son refutables. ${ }^{21}$}

5. El conocimiento científico progresa y tiende a la verdad cuando se somete a crítica ${ }^{22}$ y se refutan críticamente sus conjeturas. El conocimiento científico se desarrolla con el método crítico de ensayar y suprimir errores para elegir la mejor teoría entre varias rivales a través del proceso de contrastación (Popper, 1992:38).

6. Las ciencias, al igual que la filosofía, progresan cuando se ejerce una actitud racional.

7. La actividad científica tiene una intencionalidad, se sustenta en un objeto de estudio con lenguajes descriptivos e hipótesis especializadas con expectativas de anticipación.

\section{La relación entre la observación y la teoría.}

El filósofo austríaco desarrolla una crítica a la concepción de la percepción y observación del positivismo lógico. Refuta la tesis de que la ciencia es producto de un proceso inductivo que parte de la observación, la recopilación de datos, los registros y la información sobre la naturaleza.

La ciencia no parte de recopilaciones, percepciones, observaciones $\mathrm{u}$ hechos sino de problemas... Las teorías o problemas siempre van delante (teoría o expectativa rudimentarias), preceden a la observación... partimos no de observaciones, sino de problemas prácticos o teóricos... de expectativas o refutaciones teóricas o prácticas. Todo problema surge del descubrimiento de que algo falla en nuestro presunto saber (Popper, 1994: 92; 1992:304-306).

La observación no es solo un acto fisiológico de percibir o registrar cosas, sino que está mediado por conjeturas, problemas e intereses del observador. Para demostrar esto, Popper propone a un auditorio que observe algo. A lo que le preguntan: "¿qué hay que observar?”. Con este ejemplo el filósofo quiere mostrar que la observación está focalizada por un problema, una expectativa teórica o práctica del investigador.

La percepción u observación no es un fenómeno meramente fisiológico-cerebral, sino que es, además, un mecanismo intelectual. La observación es un fenómeno mediado por artefactos teóricos, categorías conceptuales, criterios metodológicos y problemas que focalizan la mirada en la expectativa de solución teórica o práctica que tenga en manos el investigador.

En este sentido, la percepción es el producto de:

$\checkmark \quad$ El descubrimiento de una contradicción interna en nuestro presunto conocimiento; es decir, por inconsistencias de relaciones lógicas entre las teorías.

$\checkmark$ Las dificultades teóricas, experimentales o prácticas que se tienen en mente.

$\checkmark \quad$ Las conjeturas críticas que se asumen en la solución de un problema. 
$\checkmark$ Las soluciones (las que resisten las críticas más rigurosas e ingeniosas) que plantean dificultades y problemas que exigen nuevas miradas.

$\checkmark$ La capacidad creadora del investigador para encontrar soluciones a problemas no resueltos.

\section{Aportes del racionalismo crítico de Popper al estudio de la filosofía de las ciencias sociales.}

Popper se interesa por la epistemología y metodología de las ciencias sociales en diferentes apartados de su obra.

Según Gómez (2003:61) hace interesantes contribuciones en este ámbito en cuanto a la concepción de las leyes (recordemos su famosa diferenciación entre leyes universales y tendencias corrigiendo a S. Mill y oponiéndose al historicismo); la explicación de la causalidad (su interpretación propensivista de la probabilidad abre la puerta a una interpretación probabilística de la causalidad) y al problema del método (lógica) científica en ciencias sociales ${ }^{23}$.

En este sentido, a partir de la metáfora de las nubes y los relojes, Popper expone en La lógica de las ciencias sociales una teoría situacional para el análisis de los fenómenos sociales con que elabora una explicación comprensiva de la toma de decisiones de forma racional, basado en veintidós tesis. Estas tesis son argumentos valiosos para pensar la práctica científica en el contexto social.

Popper interpreta el funcionamiento regular e irregular de la realidad de los tres mundos a través de la metáfora de las nubes y los relojes.
Las nubes representan los sistemas físicos que como los gases son altamente irregulares, desordenados y más o menos impredecibles... los relojes de péndulos representan el funcionamiento regular, ordenado de los sistemas físicos el cual se puede predecir. (Popper, 1994:249). [En la mitad de estos dos extremos el de la total plasticidad y el de la regularidad está el mundo de las acciones humanas reguladas por instituciones humanas]

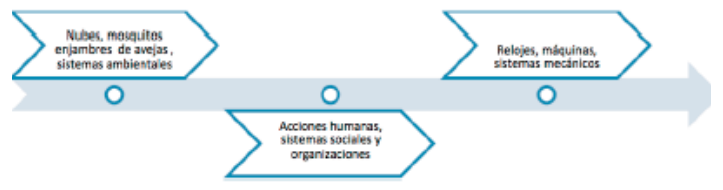

Gráfica 2. Representación del funcionamiento de las nubes y los relojes según Popper. Fuente: Propia.

Esta metáfora es la representación de es contínuum de cosas que componen el mundo en que vive y habita el ser humano. La dependencia del ser humano al medio social es muy fuerte. En vez de hablar naturaleza humana se debe reconocer dimensión social; las instituciones sociales y las tradiciones culturales en contexto inciden en el ser humano en la toma de decisiones de forma racional.

Popper, según Gómez (2003:64) apoyado en Marx sostiene la influencia del entono social en el hombre, pero al mismo tiempo que ella es creación de él; especialmente las instituciones y tradiciones culturales que las recibe y recrea a través de la educación, la información las cuales recibe de generación a generación. Pero estas estructuras si bien controlan colectiva e individualmente al hombre; este tiene la voluntad y capacidad de transfórmalas. Nuestras ideas y estructuras dependen del contexto y otros factores situacionales en que se vive o habita pero se transforma por la crítica racional. Estamos influenciados 
por el medio institucional, Pero abiertos al cambio, en este sentido, no somos autómatas determinados o cerrados al entorno.

Resalta Gómez (2003:63) que las acciones están condicionadas por el medio social, transcurre en un contexto institucional dentro del cual encontramos otros que a la manera de círculos concéntricos, delimitan el espacio y el tiempo de las acciones humanas. Finalmente hay que contar con las instituciones sociales porque son estas las que determinan el carácter social real de nuestro entorno humano.

El hombre, por ser parte de la "trama de la vida" (Capra: 1998:2) es un ser -bio-psicocultural-ambiental creador de instituciones humanas con las cuales satisface múltiples necesidades existenciales y sociales.

Hay una porción del mundo real, hechos objetivos, que son producto de los acuerdos humanos: las instituciones... sin embargo, hay otra porción del mundo real, hechos objetivos, que existen independientemente de cualquier opinión, representación, voluntad o lenguaje humano como son: la lluvia, las nubes, el hielo o el Everest. (Searle, 1997:22-24).

Pero, ¿qué son las instituciones humanas? Según, Searle (1997:45) son un conjunto de reglas constitutivas y regulativas de patrones colectivos que coordinan e integran el actuar colectivo de las personas en la sociedad. Algunas reglas regulan actividades; pero otras no solo las regulan, sino que las constituyen. Ellas son similares a las reglas del ajedrez, que no solo regulan el juego previamente, sino que lo constituyen; de suerte que si no se sigue las reglas, no se juega al ajedrez.
Las instituciones permiten realizar a los seres humanos procesos de interacción consigo mismo, con otros seres vivos, con el mundo natural, social y ambiental. Son instituciones humanas el lenguaje, el matrimonio, el dinero, el estado, la ciencia, la empresa, la universidad, el fútbol, entre otros.

En La lógica de las ciencias sociales el filósofo define que las ciencias sociales son un conjunto de problemas definidos y lógicamente diferenciados que se resuelven a partir del método de la crítica objetiva basado en la lógica situacional que estudian el entorno social e institucional en que vive el ser humano.

El filósofo austríaco propone los siguientes criterios metodológicos o reglas para el análisis crítico de los acontecimientos humanos:

1.4 Criterios lógicos: permiten el análisis de las proposiciones que dan fuerza explicativa y claridad en la búsqueda de la verdad.

\subsection{Criterios de distinción de los} intereses: están relacionados con los intereses en la búsqueda de la verdad o los que no pertenecen a ella; están presentes en el conocimiento científico de las escuelas de pensamiento de las comunidades científicas o de los científicos investigadores.

\subsection{Criterio de análisis de los problemas: el conocimiento en} ciencias sociales se desarrolla con teorías, problemas y métodos críticos que crean explicaciones comprensivas e interpretativas de las ideas o representaciones sociales, de los entornos socioculturales (familia, lenguaje, instituciones sociales), de nichos ambientales y de acciones planificadas. 
1.7 Criterios de valores científicos: la pertinencia, la relevancia, el interés, el significado, la utilidad, la fuerza explicativa, la simplicidad y la decisión son valores imposibles de eliminar en la actividad científica. Para el austríaco los valores científicos y extra-científicos no se pueden eliminar en la actividad investigativa de la ciencia. Los motivos, los ideales y la búsqueda de la verdad objetiva están profundamente anclados en los juicios de valor científico, religioso, estético y tecnológico, los cuales inciden en la investigación del mundo: sin pasión es imposible conseguir algo.

\subsection{Criterios}

metodológicos

del análisis situacional: la lógica situacional permite una reconstrucción teórica razonable y simplificada de un modelo o estructura comprensiva del funcionamiento de la realidad social. Los análisis situacionales son racionales, empíricos y susceptibles de mejoramiento.

Al igual que las ciencias naturales el método en las ciencias sociales radica en ensayar posibles soluciones para los problemas, de los cuales parten nuestras investigaciones... en el caso de que en un ensayo de solución no sea susceptible de crítica objetiva se excluye por no ser científico; aunque sólo sea provisional. Toda crítica consiste en intentos de refutación, esto es lo que le da al conocimiento objetividad científica... la crítica es lo que le da objetividad y reconocimiento en la comunidad cientifica y entre las escuelas de pensamiento... solo hay ciencia si teorizamos; esto vale también para las ciencias sociales. (Popper, 1994:94-107).
Los anteriores argumentos de Popper (1992:328) son valiosos para valorar el estatuto de las estructuras teóricas de las ciencias sociales en cuanto que dan criterios filosóficos (ontológicos, epistémicos y pragmáticos) basado en las funciones del lenguaje humano, especialmente la argumentativa, en cuanto es susceptible de crítica objetiva.

Es significativo resaltar que la lógica de la situación es, según, Popper $(1962 ; 118)$ el método adecuado para las ciencias sociales ya que frente a la influencia del Hegelianismo con los métodos esencialistas y a los métodos psicológicos dominantes en los debates epistemológicos de las ciencias sociales. La lógica situacional es un método contrastable empíricamente y refutable, según el proceder científico; puesto que los criterios o reglas de análisis situacionales son racionales, empíricamente criticables y susceptibles de mejoramiento.

La explicación de los acontecimientos sociales basados en lógicas situacionales explica la ocurrencia del comportamiento humano en conjeturas o hipótesis a partir del principio de racionalidad que interpreta la regularidad nomológica deductivo del acontecer humano.

\section{Influencia del racionalismo crítico en los estudios organizacionales y administrativos en la literatura especializada colombiana.}

Existe una estrecha relación y diferencia entre instituciones y organizaciones. Aquellas son un cuerpo de reglas que instituyen tradiciones jurídico- culturales que ordena, legitiman y normalizar el comportamiento de las personas, los grupos, las organizaciones y de la sociedad. Mientras que las organizaciones son entes sociales que, al igual que el ajedrez, están instituidas por reglas, pero no se reducen a ellas. En las organizaciones las personas, 
los grupos interactúan con reglas; pero en ellas existen otros elementos constitutivos que la hacen diferente a las instituciones.

El lenguaje humano ${ }^{24}$, institución de instituciones, no solo simboliza el mundo, sino que también es recinto que posibilita la interacción colectiva en las organizaciones a nivel individual, grupal, organizacional, sociocultural y ambiental (ecológico). La función plástica del lenguaje ${ }^{25}$, como modeladora y coordinadora de las acciones humanas es dispositivo pragmático clave para explicar los discursos sobre administración como una praxis de teorización de un saber disciplinar que estudia la dirección o gobierno de las organizaciones en el contexto de las ciencias ${ }^{26}$, las tecnologías y las humanidades.

A las funciones del lenguaje (expresiva, señaladora, descriptiva, argumentativa) de simbolizar cognoscitivamente el mundo social se debe incluir la función pragmática con la cual se puede sustentar teóricamente la función administrativa en el contexto de las ciencias sociales y de las tecnológicas administrativas relacionadas con la habilidades de planificación, coordinación, integración y comunicación en la gestión de las organizaciones.

Al valorar el problema de la naturaleza de las teorías de la administración en el estado del arte de la tesis doctoral en revistas especializada colombiana se encontró que algunos documento oficiales de la Asociación Colombiana de Facultades de Administración (ASCOLFA), de congresos y revistas de administración, emerge con claridad la influencia del racionalismo crítico en el debate epistémico de la administración.

Basado en las tesis popperianas de la función del lenguaje humano y de la lógica de las ciencias sociales es importante examinar ¿Cuál es la naturaleza de las teorías administrativas y los criterios de validez? ¿Qué alcance conviene concederle con exactitud?" (Bruyne, 1973:293).

En los diferentes artículos analizados en las revistas indexadas de administración sobre la polémica de la naturaleza de las estructuras teóricas de la administración aparecen tres perspectivas de argumentación: la del positivismo lógico iniciada por el argentino Klirskberg en los años setenta del siglo XX; la del racionalismo crítico basado la lógica de las ciencias sociales y la Kuhniana.

Castaño (2007:1), basado en la teoría situacional de Popper, ubica la administración en el campo de las ciencias sociales como un saber aplicado. Argumenta que el objeto, los métodos de trabajo disciplinar de la gestión de la organización empresarial se explican mejor desde el racionalismo crítico que desde el positivismo lógico de Carnap.

Por otro lado, Salinas (2001:7374) argumenta que el racionalismo crítico aviva en los círculos académicos del país la investigación la cientificidad de la administración como un tema de gran discusión de si la administración es ciencia, técnica o arte... el estudio de este problema permite acotar un campo de formación (profesional, laboral $\mathrm{y}$ disciplinar) en el que un egresado podrá desempeñarse socialmente con calidad. Además, permite esclarecer cuál es la naturaleza y validez científica de sus teorías... El análisis de esta problemática permite crear metodologías teóricas que coadyuvan a esclarecer el estatuto epistémico de este saber y conformar una comunidad académica critica que proporciona instrumentos conceptuales, 
metodológicos y formas de trabajo intelectual relacionada con la praxis gerencial....

Las estructuras teóricas de la administración son redes, esquemas o estructura que interpretan la gestión del mundo de las organizaciones a partir de conceptos principios, enunciados o conjeturas científicas que guían las acciones de cooperación humana. Las teorías de la administración son modelos y redes conceptuales que explican e interpretar la toma de decisiones (administrativas) en los institucionales y organizacionales en el contexto de las ciencias y las tecnologías.

En el saber administrativo existen estructuras teóricas basada en la función del lenguaje que modelan las acciones colectivas apoyadas en principios ${ }^{27}$ generales con pretensión de validez para todas las organizaciones con criterios pragmáticos.

La administración es un saber social que estudia la praxis de cooperación en el trabajo humana que se expresan objetivamente en teorías, metodologías, técnicas, instrumentos, valores, principios y habilidades del pensamiento con que se forman comunidades disciplinares y profesionales. Por eso, desde Popper las teorías de la administración interpretan el mundo humano organizacional, no el natural.

Las teorías administrativas son proposiciones que no son falsas o verdaderas; ni axiomáticas $\mathrm{o}$ leyes, según la ciencia clásica; sino que son modelos de acciones con pretensiones de ser correctas $o$ incorrectas $y$ eficaces que llevan al éxito o al fracaso en la toma de decisiones, apoyados en informaciones, en experiencias con tino o phronesis en la percepción de la realidad organizacional. (Bruyne, 1973:303).

\section{Conclusiones}

Al valorar el racionalismo crítico con respecto a las tesis del positivismo lógico se concluye que es un avance teórico y metodológico valioso en el debate epistémico de la naturaleza de las teorías de la administración ${ }^{28}$ porque:

$\checkmark$ Proporciona criterios filosóficos (ontológicos, epistémicos y Praxeológicos) para el estudio del pensamiento administrativo a partir de las funciones del lenguaje humano: el argumentativo y el pragmático.

$\checkmark \quad$ En la obra de Popper se desarrolla una noción de ciencia, sean teóricas, empíricas o prácticas, como lenguajes simbólicos cognoscitivos, argumentativos y críticos compuestos por sistemas teóricos y metodológicos que parten en visiones filosóficas [metafísicas, ontológicas, epistémicas y Praxeológicas] que orientan la búsqueda de soluciones a problemas en los tres mundos, en los que es posible sustentar una teoría de la acción o praxis en el contexto de la filosofía practica; que no es posible en el positivismo lógico por ser reduccionista y polémica en lo ontológico y epistémico .

$\checkmark$ Desarrolla criterios metodológicos que orientan la praxis científica en las ciencias sociales sobre problemas prácticos que deben someterse a la crítica objetiva rigurosa sobre cuándo y cómo las teorías son inconsistentes e incompatibles con los hechos que busca interpretar.

Proporciona una concepción de teoría como modelo, esquema o red de conceptos - diferente a la concepción 
fuerte de estructura teórica lógicomatemática del positivismo lógico - que ayuda a interpretar la gestión $\mathrm{y}$ el funcionamiento del mundo organizacional y administrativo.

\section{Referencia Bibliográfica}

Bruyne, P. (1973). Teoría Moderna de la Administración de Empresas. Madrid, España: Aguilar.

Castaño Duque, G. A. (2007). Administración y ciencia empresarial. II Congreso Internacional de Investigación en Administración. Universidad Nacional, Manizales.

Capra, Fritjof. (1998) La Trama de la Vida. $2^{\mathrm{a}}$ Edi .Barcelona-España: Anagrama.

FayoL, H. (2003). Administración Industrial y General. Bogotá, Colombia: Edigrama.

García Duque, C. E. (2001). Introducción a las lecturas de Popper. En: Cuadernos literarios -filosóficos $\mathrm{N}^{\circ} 11$. Manizales-Caldas: Universidad de Caldas.

García Duque, C. E. (1997). Evolución histórica del pensamiento científico (Desde la Antigüedad clásica hasta el periodo Moderno). Manizales: Editorial Universidad de Manizales.

Gómez Rodríguez, A. (2003) filosofía y metodología de las ciencias sociales. España, Alanza editorial.

Kuhn, T. (1992). La Estructura de las Revoluciones Científicas. México: Fondo de Cultura Económica.

Jaramillo, J. M. (2001). ¿Es la ciencia una rama de la literatura fantástica? Pretexto para una reflexión sobre el realismo. En: Cuadernos Filosófico-Literarios, 12. Manizales, Colombia: Universidad de Caldas.

Popper, K. (1962). Lógica de la investigación científica, Estructura y función o el porvenir actual de la ciencia. Madrid, España: Técnos.

Popper, K. (1967). Conjeturas y refutaciones. El desarrollo del Conocimiento Científico. Barcelona, España: Paidós.
Popper, K. (1994). En búsqueda de un mundo mejor. Barcelona, España. Paidós.

Popper, K. (2007). El Conocimiento objetivo. Madrid, España: Técnos.

Salinas Gómez, O. (2001). El discurso administrativo: Naturaleza, Objeto y Método. Memorias de ASCOLFA. Pereira-Risaralda.

Searle, J. R. (1997). La construcción de la realidad social. Barcelona, España: Paidós.

\section{Notas al lector}

1 Argumenta que el problema se ha explicado desde la teoría del monismo biológico, la teoría evolutiva y del modelo artefactos con estructuras inteligentes artificiales. La primera explicación relaciona el funcionamiento de los organismos con el uso y posesión de órganos; la segunda busca explicar el funcionamiento de los organismos desde la diferencia de los órganos de los animales y plantas más bien que el origen de los tipos específicos de conductas o tendencias comportamentales. Mientras que Popper explica el funcionamiento de los organismos similares a una máquina, a través de la teoría del computador y de la teoría del control plástico. "Tomaré como ejemplo un avión de combate -guiado por un piloto automático. El avión está construido para unos fines determinados y el piloto automático dotado de un cierto número de reacciones incorporadas en su diseño que equivale a instrucciones para atacar a un enemigo más débil y apoyar al compañero en el ataque, la defensa y esquivar a un enemigo más fuerte" (Popper, 2007:323). En Sobre las nubes y relojes desarrolla con mayores argumentos esta teoría.

2 Estas estructuras tácitas están en la mente del sujeto y son de gran interés para la psicología, mas no para la epistemología o la filosofía de la ciencia. Esta tesis es refutada por Kuhn (1992) en Estructura de las revoluciones científicas, con la noción de paradigma.

$3 \mathrm{La}$ conciencia se manifiesta o funciona como sistemas de control plástico que coordinan generalmente el comportamiento e interacción humana. Si pensamos en los sistemas de control incorporados, por ejemplo en los libros, teorías, sistemas de leyes y todo lo que constituye el universo de significados, entonces difícilmente podemos decir que la conciencia es el sistema 
de control que ocupa el puesto más elevado por esos sistemas lingüísticos exosomáticos (sistemas Peircianos de control plásticos); a su vez, esos productos o estructuras cognoscitivas objetivas inciden en la conciencia (Popper, 2007:298).

4 Popper (1994:25-28) realiza una crítica a diferentes formas de concebir la realidad. Para el enfoque clásico la realidad se compone únicamente de cosas materiales que actúan entre sí por presión, impulso, acción o contacto. En esta visión existen dos versiones: el atomismo (pequeñas partículas se entrelazan entre sí y entre una y otra existe un vacío) y el que considera que entre ellas no existe nada, son compactas. Para el enfoque moderno materialista la realidad está llena de cuerpos naturales que se mueven por fuerzas gravitacionales que actúan a distancia. El fisicalismo contemporáneo (neopositivismo lógico) explica la realidad en términos de oscilaciones, vibraciones y ondas compuestas de fuerzas y energía. Para Popper la realidad está integrada por tres mundos que interactúan entre sí.

5 La ontología es una división de la filosofía que indaga por la naturaleza de los entes y del ser que hacen parte del mundo, por las relaciones entre sí y de los supuestos o fundamentos desde los cuales se explica racionalmente la composición del mundo; y se sustenta el conocimiento. "La ontología estudia las características básicas de las entidades que en forma genérica llamamos realidad o mundo" (Jaramillo, 2001: 17).

6 No se utiliza aquí el término "mundo" para designar el universo o cosmos, sino más bien sus partes (Popper, 1994:24).

7 Popper diferencia grados de esencialismo teórico. "Aquí es donde hay que buscar la semejanza y diferencia entre mi punto de vista (la tercera concepción) del esencialismo. El filósofo realiza una crítica a la noción de teoría en Platón, Aristóteles, Descartes y Newton, que los clasifica en esencialistas de primera y segunda concepción. Niega que el objetivo de la ciencia sea el de buscar explicaciones últimas del mundo o describir las propiedades individuales o esenciales de las cosas. La ciencia interpreta las estructuras del funcionamiento del mundo natural, social y humano. La crítica la desarrolla desde el determinismo físico, psicológico y filosófico.
8 Una teoría explicativa va necesariamente más allá de un conjunto finito de enunciados contrastadores; lo mismo se puede decir incluso de una ley con un grado reducido de universalidad (Popper, 2007:22).

9 El fenómeno de la interpretación está presente en la actividad científica. Es una lectura estructurada de los fenómenos naturales.

10 Popper identifica diferentes tipos de teorías: científicas, mitológicas, metafísicas.

11 Un concepto singular hace referencia a cosas o nombres propios y los conceptos universales hacen referencia a conceptos de clase: eje, mamífero.

12 Los enunciados sintéticos se clasifican en enunciados universales y numéricamente universales. Los enunciados universales son hipótesis que tienen el carácter de leyes naturales que se refieren a acontecimientos que se dan en todo espacio y tiempo; los enunciados numéricamente universales específicos se refieren a explicaciones de acontecimientos que se dan en ciertas regiones del espacio y del tiempo. (Popper, 1962:61).

13 Popper define los enunciados existenciales como no empíricos o metafísicos, porque definen la existencia de propiedades que no están limitadas a un espacio y tiempo determinados.

14 Las explicaciones científicas en términos de leyes han de ser contrastadas independientemente en todo lugar y tiempo.

15 Un juicio es independiente cuando no es circular ni ad hoc. Popper da varios ejemplos de esta clase de argumentos.

16 Popper afirma que desde la lógica (inductiva o deductiva) no se logra explicar el avance de las teorías de Newton con respecto a las de Kepler y Galileo, solo el ingenio de Newton puede mostrar profundidad de las teorías en las que los planteamientos de aquellos astrónomos son evidencia de lo que plantea Newton. No es una conjunción de ambas posturas, es una corrección y explicación de ambas teorías (Popper, 2007:243).

17 El uso del término modelo es polisémico $\mathrm{y}$ ambivalente. Algunas veces se entiende 
como estructura lógico-matemática que articula coherentemente diferentes variables con las que se explica un fenómeno; también como un algoritmo que simula el flujo de información; como la representación interna de la estructura de una teoría; también se entiende aquello a partir de lo cual se hace una pintura o un retrato; como maqueta que representa en pequeña escala un artefacto; o modelo es una estructura lingüística que describe analógica o metafóricamente un fenómeno natural, social o humano.

18 La explicación es un conjunto de enunciados mediante los cuales se describe el estado de la cuestión que se va a explicar (el explicandum), sirviéndose para ello de otros enunciados; los enunciados explicativos constituyen la explicación en sentido estricto (el explicans del explicandum) (Popper, 1992:231).

19 El método son reglas de juego que guían la creación e innovación del conocimiento científico. Son diferentes a las reglas de la lógica y la lingüística.

20 Con esta tesis y apoyado en García (2001:5) se fortalece una polémica de la naturaleza de las teorías y su relación con el mundo natural, plateados por los realistas científicos, los convencionalista e instrumentalistas y con la teoría evolucionista de las ciencias. Este problema no se desarrolla pero es clave en filosofía de la ciencia contemporánea.

21 La demarcación es un criterio para determinar el grado de cientificidad de las teorías a partir del principio de refutabilidad o testabilidad, que permite identificar cuándo los enunciados son empíricos o pseudocientíficos, o si son otra clase de enunciados: religiosos, metafísicos (Popper, 1967:23). Un enunciado es empírico si hay conjunciones (finitas) de enunciados singulares (enunciados básicos o contrastadores) que lo contradigan (Popper, 2007:28). Los enunciados contrastadores permiten justificar empíricamente las teorías y elegir entre las mejores. Con este mecanismo se explica el progreso científico.

22 Popper (1967:77) clasifica las actitudes ante el conocimiento de dogmático, pseudocientífico y científico o crítico.
23 Con la tesis de la lógica situacional se opuso a los planteamientos de la escuela de Frankfort (conocida como la disputa del positivismo en la sociología alemana) y al holismo metodológico del historicismo y sociología marxista, especialmente relacionada con los hechos sociales.

24 El lenguaje tiene primacía sobre las demás instituciones .Desde este punto de vista, el lenguaje es la institución humana social básica en cuanto que todas las demás lo presuponen; pero el lenguaje, no las presupone a ellas. Usted puede tener lenguaje sin tener dinero, matrimonio, universidad; pero no al revés (SEARLE; 1997: 76).

25 Según Popper las estructuras cognoscitivas objetivas del lenguaje humano cumplen diferentes funciones (expresiva, señaladora, descriptiva y argumentativa), que son claves para la resolución de problemas en las ciencias, las humanidades y la filosofía. De igual forma, se puede argumentar, que las estructuras cognoscitivas objetivas del lenguaje humano (las estructuras teóricas) desempeñan, también, la función creadora, coordinadora, comunicativa y directiva en las organizaciones.

26 La ciencia que también es una institución social está reglamentada por un conjunto de reglas que define criterios de cuándo y cómo un saber disciplinar es considerado como científico.

27 Fayol, padre la administración moderna, propone 14 principios.

28 Este problema se desarrolla en la investigación de la tesis de maestría y de la tesis doctoral. 